\title{
Numerical implementation of local effects due to two-dimensional discontinuous loads using special elements based on boundary integrals
}

\author{
Hui Wang ${ }^{\mathrm{a}}$, Qing-Hua Qin ${ }^{\mathrm{b}, *}$ \\ a Institute of Scientific and Engineering Computation, Henan University of Technology, Zhengzhou 450052, PR China \\ ${ }^{\mathrm{b}}$ Research School of Engineering, Australian National University, Canberra, ACT 0200, Australia
}

\section{A R T I C L E I N F O}

\section{Article history:}

Received 17 October 2011

Accepted 13 June 2012

Available online 20 July 2012

Keywords:

Special-purpose element

Discontinuous loads

Fundamental solutions

Hybrid finite element method

Plane elasticity

\begin{abstract}
A B S T R A C T
In this paper, special-purpose elements are developed for solving local effects caused by discontinuous loads such as concentrated forces, line loads and patch loads applied in plane elastic structures. During the derivation of the special-purpose elements, the interior displacement and stress fields are composed of two parts: (1) the homogeneous solution part, which is represented by a linear combination of fundamental solutions at a number of source points outside the element domain; and (2) the particular load-dependent part, which is analytically represented by suitable local solutions. Meanwhile the independent frame displacements defined over the element boundary are approximated by conventional shape functions. The linkage between the two independent fields is established through use of a newly constructed hybrid variational functional, in which discontinuous loads are treated as generalized body forces. Using the property of delta function, the domain integral associated with discontinuous loads in the variational functional can be removed. The advantage of such specialpurpose elements is that a large element, independent of the location of discontinuous loads, can be used to avoid the requirement of mesh refinement in the vicinity of the area with local loads. Numerical experiments are carried out to verify the special-purpose elements and to investigate their effectiveness in terms of mesh reduction and accuracy.
\end{abstract}

(c) 2012 Elsevier Ltd. All rights reserved.

\section{Introduction}

It is important for researchers to accurately predict the deformation behavior of elastic bodies due to local stress singularities induced by discontinuous loads, such as point loads, line loads, and patch loads. In practice, the treatment of discontinuous loads is problematic for numerical simulation. For example, an extremely refined mesh is usually required to capture the dramatic variations of displacement and stress in the vicinity of discontinuous loads when the conventional finite element method (FEM) [1] is employed. Moreover, among boundary-type methods such as the boundary element method (BEM) $[2,3]$, it is also inconvenient to deal with multiple internal discontinuous loads in elastic structures by integral equations established in the entire domain.

As an alternative to the conventional FEM and BEM, a formulation of the hybrid FEM based on fundamental solutions, known as HFS-FEM, has recently been presented [4] and subsequently applied to plane isotropic and orthotropic elastic problems with or without hole defects [5-7], simulation of

\footnotetext{
* Corresponding author.

E-mail address: qinghua.qin@anu.edu.au (Q.-H. Qin).
}

functionally graded material with a graded element [8], as well as heat transfer in the human eye and composites $[9,10]$. This method differs from the hybrid stress boundary element method (HSBEM) based on the Hellinger-Reissner potential and interior stress approximation with summation of fundamental solutions $[11,12]$ and the hybrid Trefftz FEM (HT-FEM) using truncated Trefftz solutions as element interior interpolation kernels [13-15]. The proposed HFS-FEM is based on two-field approximations, one being intra-element displacement and stress fields exactly satisfying the governing partial differential equations of the problem of interest, and the other a conforming frame field of displacements defined along the element boundary through which the inter-element continuity and boundary conditions are enforced by the hybrid functional in a weak sense. The final unknowns in the stiffness equation are the usual nodal displacements of the frame field. This feature allows us to construct arbitrarily shaped polygonal elements and special-purpose elements such as circular or elliptic hole elements [6,7] and graded elements [8], using corresponding special fundamental solutions of the problem under consideration. As a result, significant mesh reduction can be achieved with acceptable numerical accuracy.

The purpose of this paper is to develop special elements for handling local effects caused by discontinuous loads such as 
point, line, and patch loads imposed inside the solution domain. In the proposed hybrid finite element formulation, the intraelement fields are augmented with suitable local singular solutions corresponding to the discontinuous loads of interest, so that they can accurately capture the dramatic variations of displacement and stress fields near the locations at which the discontinuous loads are applied. Consequently, mesh refinement is not required around areas with local singularities and large polygonal special-purpose elements can be constructed to enclose the discontinuous loads. In this study, local singular solutions related to discontinuous loads are obtained by means of the physical definition of fundamental solutions. The performance of the proposed special-purpose elements is assessed by comparison with that of analytical solutions or conventional FEM solutions.

The paper is arranged as follows. Section 2 provides a short description of plane elastic theory. In Section 3, local solutions for point, line, and patch loads are, respectively, derived using a fundamental solution approach. The application of local solutions to generate special-purpose elements is discussed in Section 4. Section 5 presents some numerical examples for assessing the proposed method. Some concluding remarks are made in Section 6.

\section{Basic equations of plane elasticity}

In this section, plane elastic theory is briefly reviewed to establish the notation and formulation used in later sections. We consider a well-posed elastic problem in a domain denoted by $\Omega$ bounded by its boundary $\Gamma$. The corresponding partial differential governing equations and boundary conditions are, respectively, given by [16]

$$
\left.\begin{array}{l}
\sigma_{i j, j}+b_{i}=0 \\
\varepsilon_{i j}=\frac{1}{2}\left(u_{i, j}+u_{j, i}\right) \\
\sigma_{i j}=\lambda \delta_{i j} \varepsilon_{k k}+2 \mu \varepsilon_{i j}
\end{array}\right\} \quad \text { in } \Omega
$$

and

$$
\left.\begin{array}{ll}
u_{i}=\bar{u}_{i} & \text { on } \Gamma_{u} \\
s_{i}=\sigma_{i j} n_{j}=\bar{s}_{i} & \text { on } \Gamma_{s}
\end{array}\right\}
$$

where a comma denotes partial differentiation and the Einstein summation convention over repeated indices is used to make the expressions compact. $\sigma_{i j}$ is the stress tensor, $b_{i}$ is the body force component, $\varepsilon_{i j}$ denotes the elastic strain tensor, $u_{i}$ is the displacement field component, and $\delta_{i j}$ is Kronecker's delta. $\lambda$ and $\mu$ are, respectively, the Lamé elastic constants and can usually be expressed in terms of Young's modulus $E$ and Poisson ratio $v$ as

$\lambda=\frac{3-\kappa}{\kappa-1} \mu, \quad \mu=\frac{E}{2(1+v)}$

with $\kappa=3-4 v$ for plane strain state and $\kappa=(3-v) /(1+v)$ for plane stress state. Also, $\bar{u}_{i}$ and $\bar{s}_{i}$ are imposed boundary displacement and traction components, respectively, $\Gamma=\Gamma_{u} \cup \Gamma_{q}$ is the boundary of the solution domain $\Omega$, and $n_{i}$ represents the $i$ th component of the outward normal vector to the boundary $\Gamma$.

Eq. (1) can be rewritten in compact form as

$\{\lambda+\mu\} u_{j, j i}(\mathbf{x})+\mu u_{i, j j}(\mathbf{x})+b_{i}=0$

which represents the classic Navier-Cauchy equations in terms of displacement fields.

When the body force becomes a unit concentrated force applied at the point $\mathbf{x}^{s}$ in an infinite domain, the solutions of Eq. (4) are known as fundamental solutions or free space Green's functions [2,17]. This physical definition of fundamental solutions can be used in the present hybrid finite element model to construct local solutions employed in the developed special- purpose elements to deal effectively with internal discontinuous forces.

\section{Local solutions associated with local discontinuous loads}

In this study, we focus on local solutions induced by internal point, line, and patch loads, as displayed in Fig. 1. All these internal loads can be regarded as generalized body forces from the physical point of view, such that their effects can be represented by the fundamental solutions that follow.

\subsection{Local solutions due to point loads}

We consider a plane elastic problem in an infinite domain subjected to a pair of internal concentrated forces $\left(P_{1}, P_{2}\right)$ along the directions of two coordinate axes, as shown in Fig. 2 . In this case, the concentrated forces can be regarded as generalized body forces with intensity $P_{i} \delta\left(\mathbf{x}-\mathbf{x}^{s}\right)(i=1,2)$ at the point $\mathbf{x}^{s}=\left(x_{1}^{s}, x_{2}^{s}\right)$ by using the delta function $\delta\left(\mathbf{x}-\mathbf{x}^{s}\right)$, and thus the Navier-Cauchy Eq. (4) can be written as $[17,18]$

$$
\{\lambda+\mu\} u_{j, j i}(\mathbf{x})+\mu u_{i, j j}(\mathbf{x})+P_{i} \delta\left(\mathbf{x}-\mathbf{x}^{s}\right)=0
$$

which can be solved by means of Galerkin vector or complex functions, and the general solution expression of Eq. (5) at any point $\mathbf{x}=\left(x_{1}, x_{2}\right)$ can be written as [16]

$u_{i}\left(\mathbf{x}, \mathbf{x}^{s}\right)=U_{l i}^{*}\left(\mathbf{x}, \mathbf{x}^{s}\right) P_{l}$

in which the kernel functions [17]

$U_{l i}^{*}\left(\mathbf{x}, \mathbf{x}^{s}\right)=\frac{1}{2 \pi \mu(1+\kappa)}\left\{-\kappa \delta_{l i} \ln r+\frac{r_{i} r_{l}}{r^{2}}\right\}$

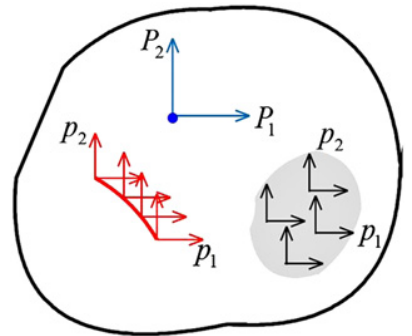

Fig. 1. Sketch of plane elastic domain under internal discontinuous loads.

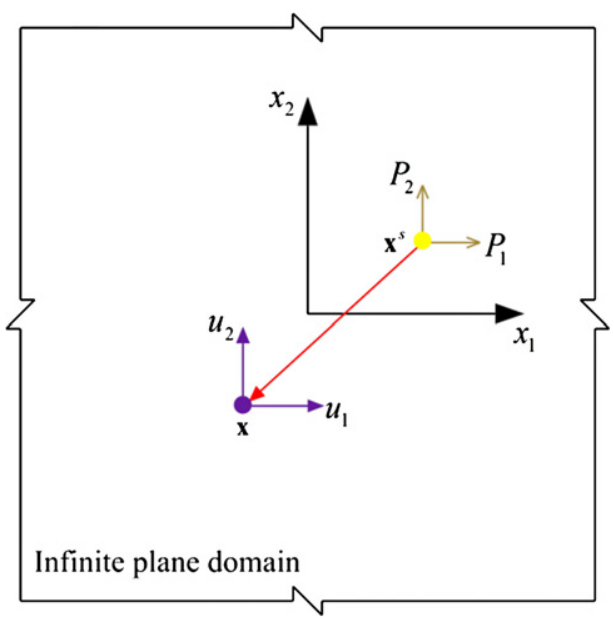

Fig. 2. Effect of concentrated forces in an infinite plane. 
are the fundamental solutions of the problems. In Eq. (7), $r$ is the distance between the points $\mathbf{x}$ and $\mathbf{x}^{s}$, i.e.,

$r^{2}=r_{i} r_{i}$ with $r_{i}=x_{i}-x_{i}^{s}$.

Substitution of Eq. (6) into the strain-displacement relation and then into the constitutive equation given in Eq. (1) yields the following local stress fields induced by the imposed point loads $\left(P_{1}, P_{2}\right)$ :

$\sigma_{i j}\left(\mathbf{x}, \mathbf{x}^{S}\right)=S_{l i j}^{*}\left(\mathbf{x}, \mathbf{x}^{S}\right) P_{l}$

with the stress fundamental solutions in the form

$S_{l i j}^{*}\left(\mathbf{x}, \mathbf{x}^{S}\right)=\frac{1}{2 \pi(1+\kappa)}\left\{\frac{(1-\kappa)\left(\delta_{l j} r_{i}+\delta_{l i} r_{j}-\delta_{i j} r_{l}\right)}{r^{2}}-\frac{4 r_{i} r_{j} r_{l}}{r^{4}}\right\}$.

Considering the physical meaning of fundamental solutions, the displacements and stresses induced by concentrated forces $\left(P_{1}, P_{2}\right)$ have the same distributions as the fundamental solutions. The stress components will vanish when the point $\mathbf{x}$ is distant from the point $\mathbf{x}_{s}$ at which the concentrated forces are applied and will be singular when the point $\mathbf{x}$ overlaps the point $\mathbf{x}_{s}$.

\subsection{Local solutions due to line loads}

For the case of arbitrarily distributed line loads, as shown in Fig. 3, with distributed load intensity $p_{1}$ and $p_{2}$, respectively, parallel to the $x_{1}$ and $x_{2}$ axes, the resultant forces on the differential element of arc length $\mathrm{d} L$ are given by

$\mathrm{d} \mathbf{P}=\left\{p_{1}\left(\mathbf{x}^{s}\right) \quad p_{2}\left(\mathbf{x}^{s}\right)\right\}^{\mathrm{T}} \mathrm{d} L$

which leads to the density of the body forces $\mathrm{d} \mathbf{b}=\mathrm{d} \mathbf{P} \delta\left(\mathbf{x}-\mathbf{x}^{s}\right)$ produced by the resultant forces $\mathrm{dP}$, and thus the generalized body forces along the curved line are given by

$\mathbf{b}(\mathbf{x})=\int_{L_{A B}} \mathrm{~d} \mathbf{b}=\int_{L_{A B}}\left\{p_{1}\left(\mathbf{x}^{S}\right) \quad p_{2}\left(\mathbf{x}^{S}\right)\right\}^{\mathrm{T}} \delta\left(\mathbf{x}-\mathbf{x}^{S}\right) \mathrm{d} L$.

Hence, the induced displacement and stress fields can be obtained by integrating the point-load solutions given in Eqs. (6) and (9) along the curved line segment $L_{A B}$ and this leads to the following line integrals with respect to arc length along the curve $L_{A B}$ :

$u_{i}(\mathbf{x})=\int_{L_{A B}} U_{l i}^{*}\left(\mathbf{x}, \mathbf{x}^{S}\right) p_{l}\left(\mathbf{x}^{S}\right) \mathrm{d} L$

$\sigma_{i j}(\mathbf{x})=\int_{L_{A B}} S_{l i j}^{*}\left(\mathbf{x}, \mathbf{x}^{S}\right) p_{l}\left(\mathbf{x}^{S}\right) \mathrm{d} L$.

If the smooth curve $L_{A B}$ can be expressed in the form $x_{2}^{s}=f\left(x_{1}^{s}\right) \quad$ for $a \leq x_{1}^{s} \leq b$ then the differential element of arc length $d L$ can be written as

$\mathrm{d} L=\sqrt{\left(d x_{1}^{S}\right)^{2}+\left(\mathrm{d} x_{2}^{S}\right)^{2}}=\Lambda\left(\mathbf{x}^{s}\right) \mathrm{d} x_{1}^{S}$

where

$\Lambda\left(\mathbf{x}^{s}\right)=\sqrt{1+\left(\frac{\mathrm{d} f}{\mathrm{~d} x_{1}^{s}}\right)^{2}}$.

Consequently, the line integrals above can be converted into general integrals in terms of the single variable $x_{1}^{s}$

$u_{i}(\mathbf{x})=\int_{a}^{b}\left\{U_{l i}^{*}\left(\mathbf{x}, \mathbf{x}^{s}\right) p_{l}\left(\mathbf{x}^{s}\right) \Lambda\left(\mathbf{x}^{s}\right)\right\} \mathrm{d} x_{1}^{s}$

$\sigma_{i j}(\mathbf{x})=\int_{a}^{b}\left\{S_{l i j}^{*}\left(\mathbf{x}, \mathbf{x}^{s}\right) p_{l}\left(\mathbf{x}^{s}\right) \Lambda\left(\mathbf{x}^{s}\right)\right\} \mathrm{d} x_{1}^{s}$

which can be evaluated by numerical integration techniques.

\subsection{Local solutions due to patch loads}

Similarly, in the case of distributed patch loads, as shown in Fig. 4, with load intensity $p_{1}$ and $p_{2}$, respectively, along the directions of two coordinate axes, the resultant forces on the differential elementary area $\mathrm{d} A$ can be written as

$\mathrm{d} \mathbf{P}=\left\{p_{1}\left(\mathbf{x}^{S}\right) \quad p_{2}\left(\mathbf{x}^{s}\right)\right\}^{\mathrm{T}} \mathrm{d} A$

which leads to the density of the body forces $\mathrm{d} \mathbf{b}=\mathrm{d} \mathbf{P} \delta\left(\mathbf{x}-\mathbf{x}^{S}\right)$ produced by the resultant forces $\mathrm{dP}$.

Integrating $\mathrm{d} \mathbf{b}$ in the area denoted by $A$ where the patch loads are imposed yields

$\mathbf{b}(\mathbf{x})=\int_{A} \mathrm{~d} \mathbf{b}=\int_{A}\left\{p_{1}\left(\mathbf{x}^{S}\right) \quad p_{2}\left(\mathbf{x}^{S}\right)\right\}^{\mathrm{T}} \delta\left(\mathbf{x}-\mathbf{x}^{S}\right) \mathrm{d} A$.

As a result, the local solutions can be obtained by integrating the point-load solutions over the patch area, i.e.,

$u_{i}(\mathbf{x})=\int_{A} U_{l i}^{*}\left(\mathbf{x}, \mathbf{x}^{S}\right) p_{l} \mathrm{~d} A$

$\sigma_{i j}(\mathbf{x})=\int_{A} S_{l i j}^{*}\left(\mathbf{x}, \mathbf{x}^{S}\right) p_{l} \mathrm{~d} A$

\section{Formulation of hybrid finite element and special-purpose elements}

In this work, if an arbitrary polygonal element $e$ is taken into consideration, as shown in Fig. 5, the hybrid variational functional $\prod m e$ at element level based on two-field approximations is given

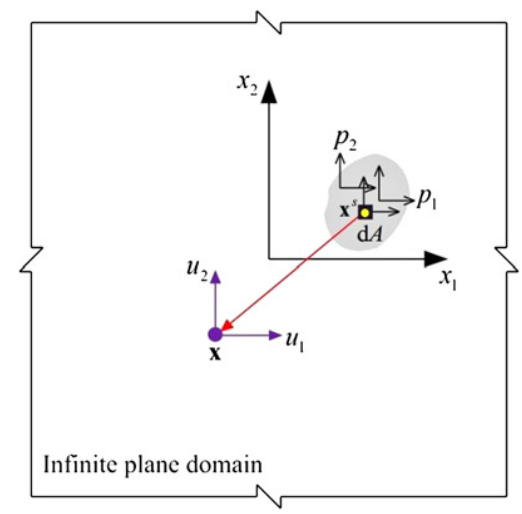

Fig. 4. Effect of patch loads in an infinite plane.

Fig. 3. Effect of local line loads in an infinite plane. 


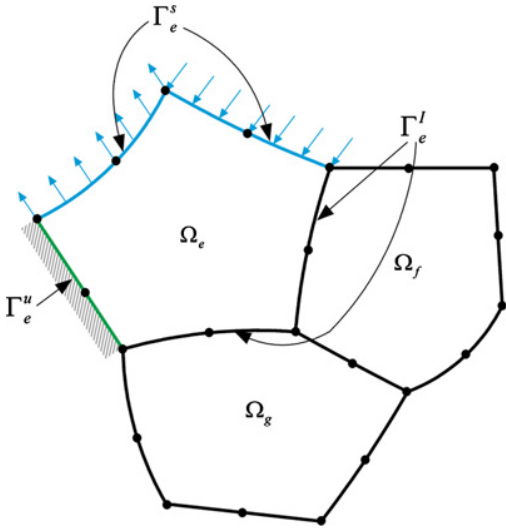

Fig. 5. Schematic of arbitrary polygonal hybrid element.

by $[5,6]$

$\Pi_{m e}=\frac{1}{2} \int_{\Omega_{e}} \boldsymbol{\sigma}^{\mathrm{T}} \boldsymbol{\varepsilon} \mathrm{d} \Omega-\int_{\Omega_{e}} \mathbf{b}^{\mathrm{T}} \mathbf{u} \mathrm{d} \Omega-\int_{\Gamma_{e}^{s}} \overline{\mathbf{s}}^{\mathrm{T}} \tilde{\mathbf{u}} \mathrm{d} \Gamma+\int_{\Gamma_{e}^{s}} \mathbf{s}^{\mathrm{T}}(\tilde{\mathbf{u}}-\mathbf{u}) \mathrm{d} \Gamma$

where $\Omega_{e}$ is the element domain under consideration and $\Gamma_{e}$ is its boundary, and

$\mathbf{u}=\left\{\begin{array}{ll}u_{1} & u_{2}\end{array}\right\}^{\mathrm{T}}, \quad \tilde{\mathbf{u}}=\left\{\begin{array}{ll}\tilde{u}_{1} & \tilde{u}_{2}\end{array}\right\}^{\mathrm{T}}, \quad \mathbf{b}=\left\{\begin{array}{ll}b_{1} & b_{2}\end{array}\right\}^{\mathrm{T}}$

$\boldsymbol{\sigma}=\left\{\begin{array}{lll}\sigma_{11} & \sigma_{22} & \sigma_{12}\end{array}\right\}^{\mathrm{T}}, \quad \boldsymbol{\varepsilon}=\left\{\begin{array}{lll}\varepsilon_{11} & \varepsilon_{22} & \gamma_{12}\end{array}\right\}^{\mathrm{T}}, \quad \mathbf{s}=\left\{\begin{array}{ll}s_{1} & s_{2}\end{array}\right\}^{\mathrm{T}}$.

In Eq. (25), u, $\boldsymbol{\sigma}$ and $\boldsymbol{\varepsilon}$ are, respectively, displacement, stress, and strain column vectors defined within the element domain $\Omega_{e}$, and $\tilde{\mathbf{u}}$ is an independent displacement vector defined along the element boundary $\Gamma_{e}$. s denotes the element traction vector, and $\overline{\mathbf{s}}$ is its specified value applied on the portion $\Gamma_{e}^{S}=\Gamma_{e} \cap \Gamma_{s}$ of the element boundary. Also in Fig. $5, \Gamma_{e}^{I}$ and $\Gamma_{e}^{u}=\Gamma_{e} \cap \Gamma_{u}$, respectively, are common boundaries of adjacent elements, for instance, elements $e$ and $f$ or $g$, and a boundary with a specified displacement constraint. For a well-posed element, we have

$\Gamma_{e}=\Gamma_{e}^{u} \cup \Gamma_{e}^{s} \cup \Gamma_{e}^{I}$

If the interior element approximations are required to exactly satisfy the governing Eq. (4), then applying the Gaussian theorem to the functional above we have the following simplified expression of the functional $[5,6]$ :

$\Pi_{m e}=-\frac{1}{2} \int_{\Gamma_{e}} \mathbf{u}^{\mathrm{T}} \mathbf{s} \mathrm{d} \Gamma-\frac{1}{2} \int_{\Omega_{e}} \mathbf{b}^{\mathrm{T}} \mathbf{u} \mathrm{d} \Omega-\int_{\Gamma_{e}^{s}} \overline{\mathbf{s}}^{\mathrm{T}} \tilde{\mathbf{u}} \mathrm{d} \Gamma+\int_{\Gamma_{e}} \mathbf{s}^{\mathrm{T}} \tilde{\mathbf{u}} \mathrm{d} \Gamma$.

In this paper, we discuss two types of element. One is the general hybrid element established with fundamental solution approximations in the absence of body forces, and the other is the special-purpose element in which the fundamental solution approximations used in the general hybrid element are augmented with suitable local solutions to accurately capture the local effects due to the discontinuous loads and then to avoid the problematic mesh refinement near the region over which the discontinuous loads are applied. In what follows, a detailed derivation of the special-purpose element is presented.

According to the definitions in Section 3, the generalized body forces corresponding to point, line, and patch loads are rewritten in a unified form as

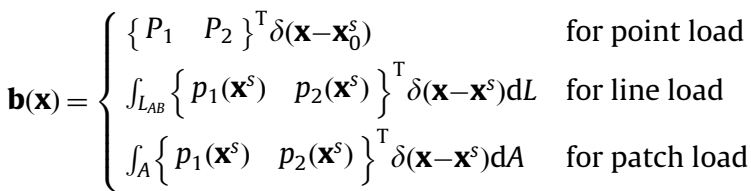

Consequently, the second integral in the variational functional (27) can be written as

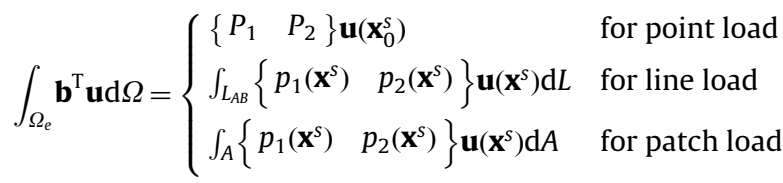

where the properties of the delta function have been employed.

If the load intensities of line and patch loads are assumed to be constant, that is, $p_{i}$ are constant, then Eq. (29) can be reduced to

$\int_{\Omega_{e}} \mathbf{b}^{\mathrm{T}} \mathbf{u d} \Omega= \begin{cases}\mathbf{P}_{0}^{\mathrm{T}} \mathbf{u}\left(\mathbf{x}_{0}^{S}\right) & \text { for point load } \\ \mathbf{P}_{0}^{\mathrm{T}} \int_{L_{A B}} \mathbf{u}\left(\mathbf{x}^{S}\right) \mathrm{d} L & \text { for line load } \\ \mathbf{P}_{0}^{\mathrm{T}} \int_{A} \mathbf{u}\left(\mathbf{x}^{S}\right) \mathrm{d} A & \text { for patch load }\end{cases}$

where

$\mathbf{P}_{0}=\left\{\begin{array}{ll}P_{1} & P_{2}\end{array}\right\}^{\mathrm{T}}$

for the case of concentrated forces, and

$\mathbf{P}_{0}=\left\{\begin{array}{ll}p_{1} & p_{2}\end{array}\right\}^{\mathrm{T}}$

for the case of distributed line or patch loads.

For the hybrid element model under consideration, it is natural to approximate the element internal displacement and stress fields by the formulation

$\mathbf{u}(\mathbf{x})=\mathbf{N}(\mathbf{x}) \mathbf{c}_{e}+\widehat{\mathbf{u}}(\mathbf{x})$

$\boldsymbol{\sigma}(\mathbf{x})=\mathbf{T}(\mathbf{x}) \mathbf{c}_{e}+\widehat{\boldsymbol{\sigma}}(\mathbf{x})$

where

$\mathbf{c}_{e}=\left\{\begin{array}{lllll}c_{11} & c_{21} & \cdots & c_{1 n_{s}} & c_{2 n_{s}}\end{array}\right\}^{\mathrm{T}}$

is the unknown coefficient vector, and

$\mathbf{N}=\left[\begin{array}{lllll}U_{11}^{*}\left(\mathbf{x}, \mathbf{y}_{1}\right) & U_{21}^{*}\left(\mathbf{x}, \mathbf{y}_{1}\right) & \cdots & U_{11}^{*}\left(\mathbf{x}, \mathbf{y}_{n_{s}}\right) & U_{21}^{*}\left(\mathbf{x}, \mathbf{y}_{n_{s}}\right) \\ U_{12}^{*}\left(\mathbf{x}, \mathbf{y}_{1}\right) & U_{22}^{*}\left(\mathbf{x}, \mathbf{y}_{1}\right) & \cdots & U_{12}^{*}\left(\mathbf{x}, \mathbf{y}_{n_{s}}\right) & U_{22}^{*}\left(\mathbf{x}, \mathbf{y}_{n_{s}}\right)\end{array}\right]$

$\mathbf{T}=\left[\begin{array}{lllll}\sigma_{111}^{*}\left(\mathbf{x}, \mathbf{y}_{1}\right) & \sigma_{211}^{*}\left(\mathbf{x}, \mathbf{y}_{1}\right) & \cdots & \sigma_{111}^{*}\left(\mathbf{x}, \mathbf{y}_{n_{s}}\right) & \sigma_{211}^{*}\left(\mathbf{x}, \mathbf{y}_{n_{s}}\right) \\ \sigma_{122}^{*}\left(\mathbf{x}, \mathbf{y}_{1}\right) & \sigma_{222}^{*}\left(\mathbf{x}, \mathbf{y}_{1}\right) & \cdots & \sigma_{122}^{*}\left(\mathbf{x}, \mathbf{y}_{n_{s}}\right) & \sigma_{222}^{*}\left(\mathbf{x}, \mathbf{y}_{n_{s}}\right) \\ \sigma_{112}^{*}\left(\mathbf{x}, \mathbf{y}_{1}\right) & \sigma_{212}^{*}\left(\mathbf{x}, \mathbf{y}_{1}\right) & \cdots & \sigma_{112}^{*}\left(\mathbf{x}, \mathbf{y}_{n_{s}}\right) & \sigma_{212}^{*}\left(\mathbf{x}, \mathbf{y}_{n_{s}}\right)\end{array}\right]$

are the coefficient matrices, $n_{s}$ is the number of source points $\mathbf{y}_{j}$ outside the element domain, as in the method of fundamental solutions (MFS) $[19,20]$. The enriched terms $\widehat{\mathbf{u}}$ and $\widehat{\boldsymbol{\sigma}}$ are corresponding local solutions related to discontinuous loads.

For the convenience of derivation, Eq. (33) is rewritten in matrix form as

$\mathbf{u}(\mathbf{x})=\mathbf{N}(\mathbf{x}) \mathbf{c}_{e}+\mathbf{N}_{0}(\mathbf{x}) \mathbf{P}_{0}$
$\boldsymbol{\sigma}(\mathbf{x})=\mathbf{T}(\mathbf{x}) \mathbf{c}_{e}+\mathbf{T}_{0}(\mathbf{x}) \mathbf{P}_{0}$

where

$\mathbf{N}_{0}(\mathbf{x})=\left[\begin{array}{cc}U_{11}^{*}\left(\mathbf{x}, \mathbf{x}_{0}^{s}\right) & U_{21}^{*}\left(\mathbf{x}, \mathbf{x}_{0}^{s}\right) \\ U_{12}^{*}\left(\mathbf{x}, \mathbf{x}_{0}^{s}\right) & U_{22}^{*}\left(\mathbf{x}, \mathbf{x}_{0}^{s}\right)\end{array}\right], \quad \mathbf{T}_{0}(\mathbf{x})=\left[\begin{array}{cc}\sigma_{11}^{*}\left(\mathbf{x}, \mathbf{x}_{0}^{s}\right) & \sigma_{211}^{*}\left(\mathbf{x}, \mathbf{x}_{0}^{s}\right) \\ \sigma_{122}^{*}\left(\mathbf{x}, \mathbf{x}_{0}^{s}\right) & \sigma_{22}^{*}\left(\mathbf{x}, \mathbf{x}_{0}^{s}\right) \\ \sigma_{112}^{*}\left(\mathbf{x}, \mathbf{x}_{0}^{s}\right) & \sigma_{212}^{*}\left(\mathbf{x}, \mathbf{x}_{0}^{s}\right)\end{array}\right]$

for point loads, and

$\mathbf{N}_{0}(\mathbf{x})=\int_{L_{A B}}\left[\begin{array}{cc}U_{11}^{*}\left(\mathbf{x}, \mathbf{x}^{s}\right) & U_{21}^{*}\left(\mathbf{x}, \mathbf{x}^{s}\right) \\ U_{12}^{*}\left(\mathbf{x}, \mathbf{x}^{s}\right) & U_{22}^{*}\left(\mathbf{x}, \mathbf{x}^{s}\right)\end{array}\right] \mathrm{d} L, \quad \mathbf{T}_{0}(\mathbf{x})=\int_{L_{A B}}\left[\begin{array}{ccc}\sigma_{111}^{*}\left(\mathbf{x}, \mathbf{x}^{s}\right) & \sigma_{211}^{*}\left(\mathbf{x}, \mathbf{x}^{s}\right) \\ \sigma_{12}^{*}\left(\mathbf{x}, \mathbf{x}^{s}\right) & \sigma_{222}^{*}\left(\mathbf{x}, \mathbf{x}^{s}\right) \\ \sigma_{112}^{*}\left(\mathbf{x}, \mathbf{x}^{s}\right) & \sigma_{212}^{*}\left(\mathbf{x}, \mathbf{x}^{s}\right)\end{array}\right] \mathrm{d} L$ 
for line loads, and

$\mathbf{N}_{0}(\mathbf{x})=\int_{A}\left[\begin{array}{ll}U_{11}^{*}\left(\mathbf{x}, \mathbf{x}^{S}\right) & U_{21}^{*}\left(\mathbf{x}, \mathbf{x}^{s}\right) \\ U_{12}^{*}\left(\mathbf{x}, \mathbf{x}^{S}\right) & U_{22}^{*}\left(\mathbf{x}, \mathbf{x}^{S}\right)\end{array}\right] \mathrm{d} A, \quad \mathbf{T}_{0}(\mathbf{x})=\int_{A}\left[\begin{array}{ll}\sigma_{111}^{*}\left(\mathbf{x}, \mathbf{x}^{S}\right) & \sigma_{211}^{*}\left(\mathbf{x}, \mathbf{x}^{S}\right) \\ \sigma_{122}^{*}\left(\mathbf{x}, \mathbf{x}^{S}\right) & \sigma_{222}^{*}\left(\mathbf{x}, \mathbf{x}^{S}\right) \\ \sigma_{112}^{*}\left(\mathbf{x}, \mathbf{x}^{S}\right) & \sigma_{212}^{*}\left(\mathbf{x}, \mathbf{x}^{S}\right)\end{array}\right] \mathrm{d} A$

for patch loads.

Furthermore, the corresponding traction vector $\mathbf{s}$ can be given by

$\mathbf{s}(\mathbf{x})=\mathbf{Q}(\mathbf{x}) \mathbf{c}_{e}+\mathbf{Q}_{0}(\mathbf{x}) \mathbf{P}_{0}$

with

$\mathbf{Q}(\mathbf{x})=\left[\begin{array}{lll}n_{1} & 0 & n_{2} \\ 0 & n_{2} & n_{1}\end{array}\right] \mathbf{T}(\mathbf{x}), \quad \mathbf{Q}_{0}(\mathbf{x})=\left[\begin{array}{lll}n_{1} & 0 & n_{2} \\ 0 & n_{2} & n_{1}\end{array}\right] \mathbf{T}_{0}(\mathbf{x})$

In order to enforce conformity on the displacement field $\mathbf{u}$, for instance, $\mathbf{u}_{e}=\mathbf{u}_{f}$ on $\Gamma_{e} \cap \Gamma_{f}$ of any two neighboring elements $e$ and $f$, an independent auxiliary inter-element frame field $\tilde{\mathbf{u}}$ is piecewise approximated along the element boundary in terms of the same nodal displacement degrees of freedom, $\mathbf{d}_{e}$. In this case, $\tilde{\mathbf{u}}$ is confined to the whole element boundary, that is

$\tilde{\mathbf{u}}(\mathbf{x})=\tilde{\mathbf{N}}(\mathbf{x}) \mathbf{d}_{e}$

where the matrix $\tilde{\mathbf{N}}$ consists of the conventional interpolating functions commonly used in the FEM and BEM [1,2].

Substituting Eqs. (36), (40) and (42) into the functional (27) produces

$$
\begin{aligned}
\Pi_{m e}= & -\frac{1}{2}\left\{\mathbf{c}_{e}^{\mathrm{T}} \mathbf{H}_{e} \mathbf{c}_{e}+\mathbf{c}_{e}^{\mathrm{T}} \mathbf{H}_{e 1} \mathbf{P}_{0}+\mathbf{P}_{0}^{\mathrm{T}} \mathbf{H}_{e 0} \mathbf{P}_{0}\right\}-\frac{1}{2} \mathbf{P}_{0}^{\mathrm{T}}\left\{\mathbf{L} \mathbf{c}_{e}+\mathbf{L}_{0} \mathbf{P}_{0}\right\} \\
& -\mathbf{d}_{e}^{\mathrm{T}} \mathbf{g}_{e}+\mathbf{c}_{e}^{\mathrm{T}} \mathbf{G}_{e} \mathbf{d}_{e}+\mathbf{d}_{e}^{\mathrm{T}} \mathbf{G}_{e 1} \mathbf{P}_{0}
\end{aligned}
$$

in which

$$
\begin{array}{ll}
\mathbf{H}_{e}=\int_{\Gamma_{e}} \mathbf{Q}^{\mathrm{T}} \mathbf{N} \mathrm{d} \Gamma & \mathbf{H}_{e 0}=\int_{\Gamma_{e}} \mathbf{Q}_{0}^{\mathrm{T}} \mathbf{N}_{0} \mathrm{~d} \Gamma \\
\mathbf{G}_{e}=\int_{\Gamma_{e}} \mathbf{Q}^{\mathrm{T}} \tilde{\mathbf{N}} \mathrm{d} \Gamma, & \mathbf{H}_{e 1}=\int_{\Gamma_{e}}\left(\mathbf{N}^{\mathrm{T}} \mathbf{Q}_{0}+\mathbf{Q}^{\mathrm{T}} \mathbf{N}_{0}\right) \mathrm{d} \Gamma \\
\mathbf{g}_{e}=\int_{\Gamma_{e}^{s}} \tilde{\mathbf{N}}^{\mathrm{T}} \overline{\mathbf{s}} \mathrm{d} \Gamma & \mathbf{G}_{e 1}=\int_{\Gamma_{e}} \tilde{\mathbf{N}}^{\mathrm{T}} \mathbf{Q}_{0} \mathrm{~d} \Gamma
\end{array}
$$

and

$\mathbf{L}=\mathbf{N}\left(\mathbf{x}_{0}^{S}\right), \quad \mathbf{L}_{0}=\mathbf{N}_{0}\left(\mathbf{x}_{0}^{S}\right)$

for point loads, and

$\mathbf{L}=\int_{L_{A B}} \mathbf{N}\left(\mathbf{x}^{S}\right) \mathrm{d} L, \quad \mathbf{L}_{0}=\int_{L_{A B}} \mathbf{N}_{0}\left(\mathbf{x}^{S}\right) \mathrm{d} L$

for line loads, and

$\mathbf{L}=\int_{A} \mathbf{N}\left(\mathbf{x}^{S}\right) \mathrm{d} A, \quad \mathbf{L}_{0}=\int_{A} \mathbf{N}_{0}\left(\mathbf{x}^{S}\right) \mathrm{d} A$

for patch loads.

The stationary conditions of the functional (43) with respect to unknown variables $\mathbf{c}_{e}$ and $\mathbf{d}_{e}$ gives

$\frac{\partial \Pi_{m e}}{\partial \mathbf{c}_{e}{ }^{\mathrm{T}}}=-\mathbf{H}_{e} \mathbf{c}_{e}-\frac{1}{2} \mathbf{H}_{e 1} \mathbf{P}_{0}-\frac{1}{2} \mathbf{L}^{\mathrm{T}} \mathbf{P}_{0}+\mathbf{G}_{e} \mathbf{d}_{e}=\mathbf{0}$ $\frac{\partial \Pi_{m e}}{\partial \mathbf{d}_{e}{ }^{\mathrm{T}}}=-\mathbf{g}_{e}+\mathbf{G}_{e}^{\mathrm{T}} \mathbf{c}_{e}+\mathbf{G}_{e 1} \mathbf{P}_{0}=\mathbf{0}$

from which we have the relation of undetermined coefficient vector $\mathbf{c}_{e}$ and the nodal displacement vector $\mathbf{d}_{e}$ as

$\mathbf{c}_{e}=\mathbf{H}_{e}^{-1}\left(-\frac{1}{2} \mathbf{H}_{e 1} \mathbf{P}_{0}-\frac{1}{2} \mathbf{L}^{\mathrm{T}} \mathbf{P}_{0}+\mathbf{G}_{e} \mathbf{d}_{e}\right)$

and the element stiffness equation representing the displacement-force relationship

$\mathbf{K}_{e} \mathbf{d}_{e}=\mathbf{f}_{e}$

In the element stiffness Eq. (50), the element stiffness matrix

$\mathbf{K}_{e}=\mathbf{G}_{e}^{\mathrm{T}} \mathbf{H}_{e}^{-1} \mathbf{G}_{e}$

is symmetrical and the equivalent nodal force vector is given by

$\mathbf{f}_{e}=\mathbf{g}_{e}+\frac{1}{2} \mathbf{G}_{e}^{\mathrm{T}} \mathbf{H}_{e}^{-1}\left\{\mathbf{H}_{e 1}+\mathbf{L}^{\mathrm{T}}\right\} \mathbf{P}_{0}-\mathbf{G}_{e 1} \mathbf{P}_{0}$.

In particular, in the absence of generalized body forces induced by discontinuous loads in the element, that is, $\mathbf{P}_{0}=\mathbf{0}$, the specialpurpose element presented reduces to the general hybrid element, and for this case, the unknown coefficient vector $\mathbf{c}_{e}$ in Eq. (49) and the right-hand side vector $\mathbf{f}_{e}$ in Eq. (52) are, respectively, simplified as

$\mathbf{c}_{e}=\mathbf{H}_{e}^{-1} \mathbf{G}_{e} \mathbf{d}_{e}$

and

$\mathbf{f}_{e}=\mathbf{g}_{e}$.

From the procedure above it can be seen that the independence of the interior and frame fields for the particular element allows us to construct arbitrarily shaped hybrid elements according to practical need. For example, Fig. 6 displays the polygonal geometry configurations of the special-purpose elements developed for three different load cases.

\section{Numerical assessments}

In this section a number of numerical examples are presented, demonstrating the implementation of the present algorithm using special-purpose elements for solving plane elastic problems involving the discontinuous loads described above. The first three examples are used to assess the performance of special-purpose elements, respectively, related to point, line, or patch loads in a square domain, and the last example is a mixed problem considering a circular plate simultaneously subjected to point, line, and patch loads, to demonstrate the effectiveness of the present approach for solving engineering problems with complex loads.

For convenience, in all examples, unless indicated otherwise, variables representing the geometry, loads, and material properties are made to be dimensionless and the elastic modulus and Poisson's ratio are assumed to be 1 and 0.3 , respectively.
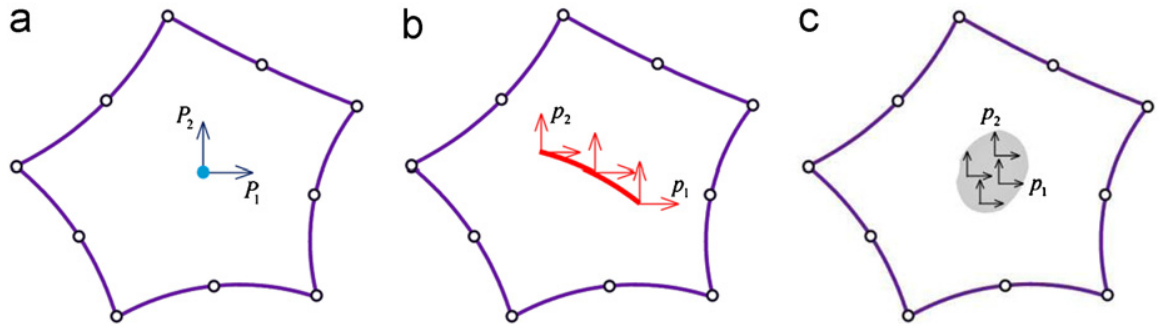

Fig. 6. Special elements associated with discontinuous loads. (a) special point-load element, (b) special line-load element, and (c) special patch-load element. 
In the present HFS-FEM, a hybrid quadrilateral general element with 8 nodes and a special-purpose element with 16 nodes are used in the following examples. We use ABAQUS to perform the conventional FEM simulation for the purpose of comparison. The element type used in ABAQUS is an 8-node quadratic quadrilateral isoparametric element.

\subsection{Local effect of point loads in a square domain}

Consider a square domain with side length 4 , subjected to a pair of concentrated forces $\left(P_{1}, P_{2}\right)$ at its center. The analytical displacement and stress solutions corresponding to the concentrated forces $\left(P_{1}, P_{2}\right)$ can be expressed in the following form by superimposing the fundamental solutions (see Eq. (7))

$$
\begin{aligned}
& u_{i}(\mathbf{x})=\frac{1}{2 \pi \mu(1+\kappa)}\left\{-\kappa P_{i} \ln \sqrt{x_{1}^{2}+x_{2}^{2}}+\frac{x_{i}\left(x_{1} P_{1}+x_{2} P_{2}\right)}{x_{1}^{2}+x_{2}^{2}}\right\} \\
& \sigma_{i j}(\mathbf{x})=\frac{1}{2 \pi(1+\kappa)}\left\{\frac{(1-\kappa)\left[P_{j} x_{i}+P_{i} x_{j}-\delta_{i j}\left(x_{1} P_{1}+x_{2} P_{2}\right)\right]}{x_{1}^{2}+x_{2}^{2}}-\frac{4 x_{i} x_{j}\left(x_{1} P_{1}+x_{2} P_{2}\right)}{\left(x_{1}^{2}+x_{2}^{2}\right)^{2}}\right\} .
\end{aligned}
$$

To keep the system solvable, Eq. (55) is also used to apply appropriately prescribed displacement boundary conditions along the outer boundary of the square domain, as shown in Fig. 7.

In our practical computation, only the case $P_{1}=0$ and $P_{2}=-1$ is assumed for the sake of simplification. One special point-load element is used to model the domain, as shown in Fig. 8(a). The

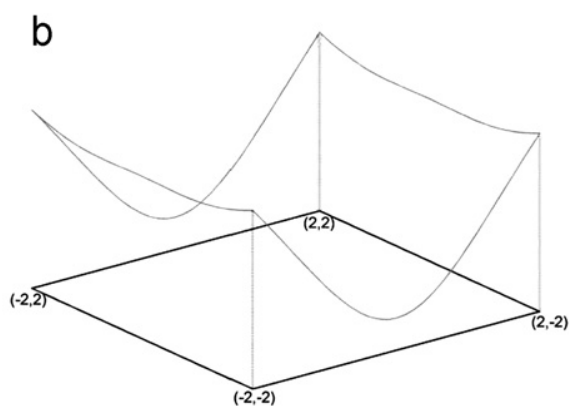

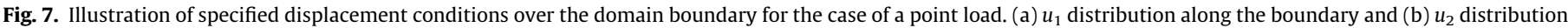
along the boundary.
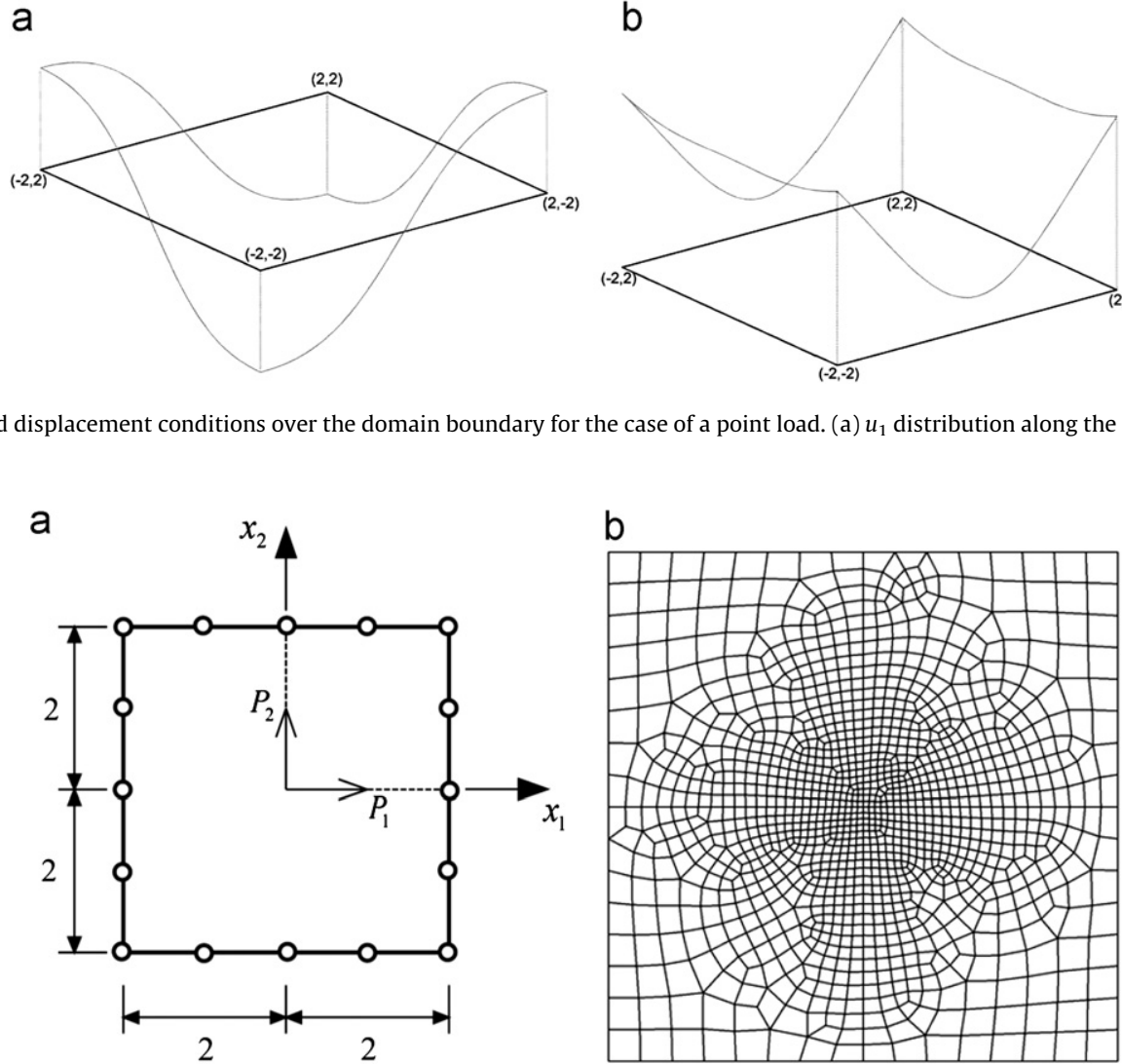

Fig. 8. (a) Schematic of square domain under centered point load and special point-load element (b) Mesh division of conventional FEM using ABAQUS
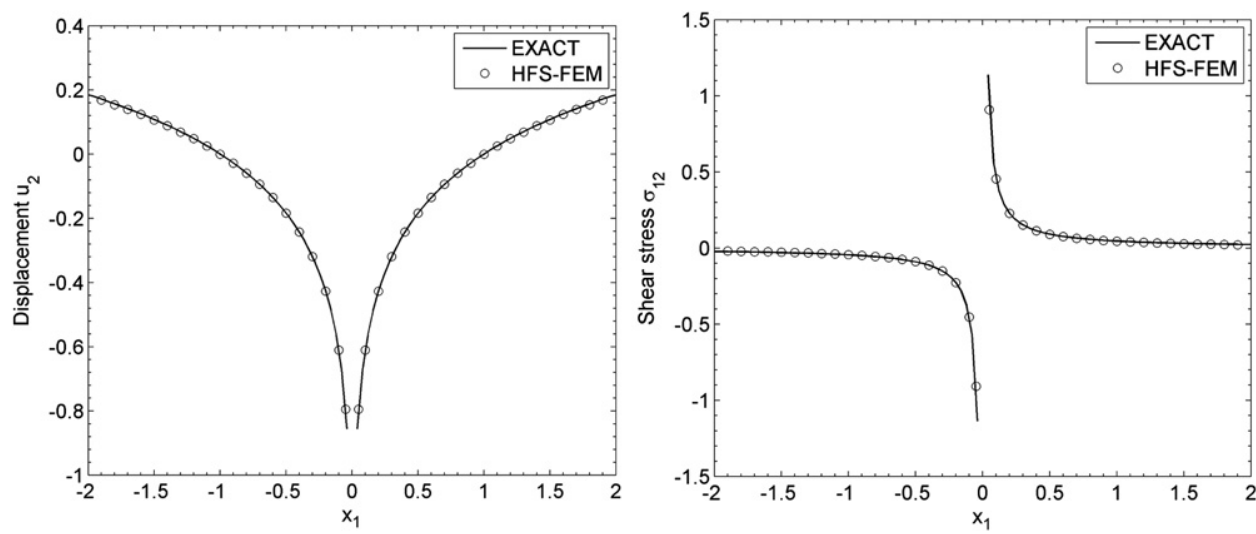

Fig. 9. Variation of displacement and stress along the $x_{1}$ axis for the case of a point load. 

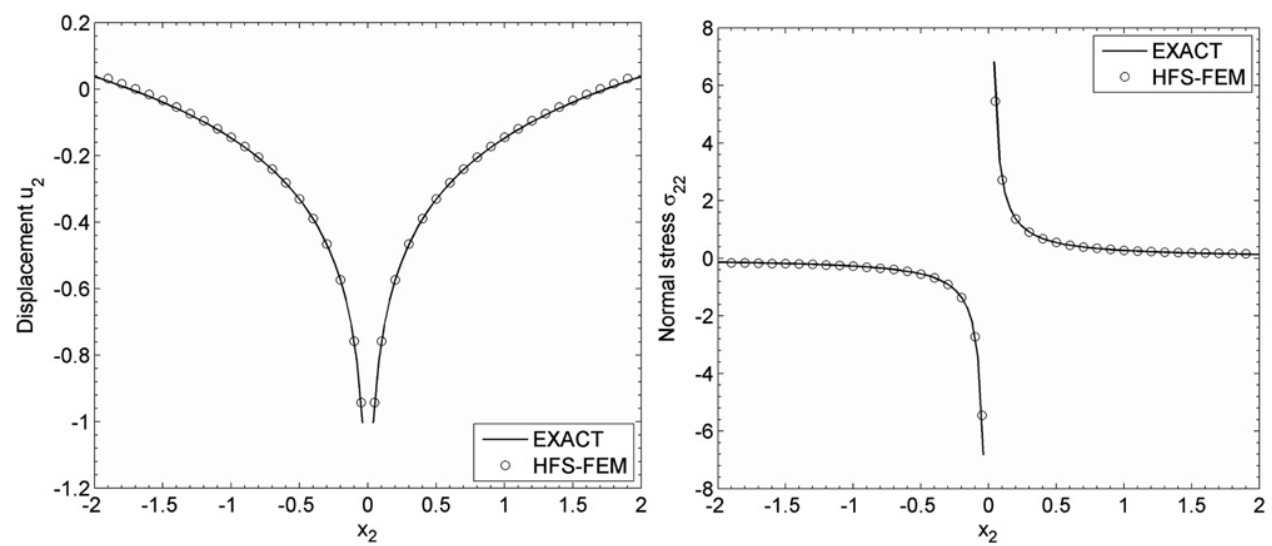

Fig. 10. Variations of displacement and stress along the $x_{2}$ axis for the case of a point load.

Table 1

Assessment of the present hybrid finite element formulation with special element for the case of a point load.

\begin{tabular}{|c|c|c|c|c|c|c|c|c|}
\hline & \multicolumn{2}{|l|}{$(0.1,0)$} & \multicolumn{2}{|l|}{$(0.05,0)$} & \multicolumn{2}{|l|}{$(0,0.1)$} & \multicolumn{2}{|l|}{$(0,0.05)$} \\
\hline & $u_{2}$ & $\sigma_{12}$ & $u_{2}$ & $\sigma_{12}$ & $u_{2}$ & $\sigma_{22}$ & $u_{2}$ & $\sigma_{22}$ \\
\hline Analytical solutions & -0.6125 & 0.4547 & -0.7969 & 0.9095 & -0.7603 & 2.7284 & -0.9447 & 5.4567 \\
\hline HFS-FEM with single special element & -0.6111 & 0.4545 & -0.7955 & 0.9093 & -0.7589 & 2.7286 & -0.9433 & 5.4569 \\
\hline ABAQUS with refined mesh & -0.6198 & 0.2654 & -0.8225 & -0.7758 & -0.7656 & 2.6543 & -0.9102 & 5.1734 \\
\hline ABAQUS with coarse mesh & -0.6327 & -0.3944 & -0.7982 & 2.3556 & -0.7233 & 2.5167 & -1.0228 & 5.5757 \\
\hline
\end{tabular}

a

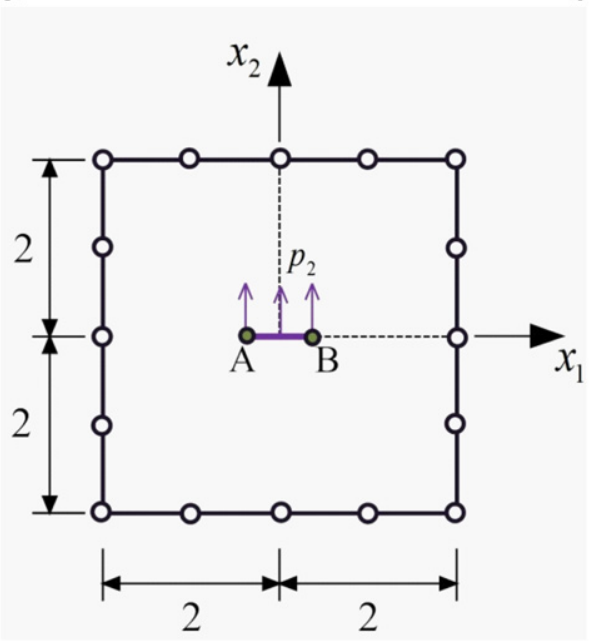

b

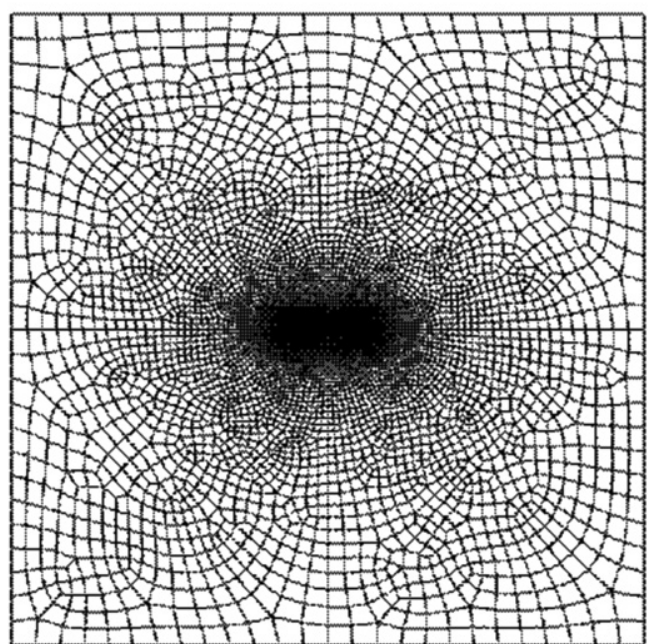

Fig. 11. (a) Sketch of square domain under a straight line load and special line-load element (b) Mesh division in conventional FEM using ABAQUS.
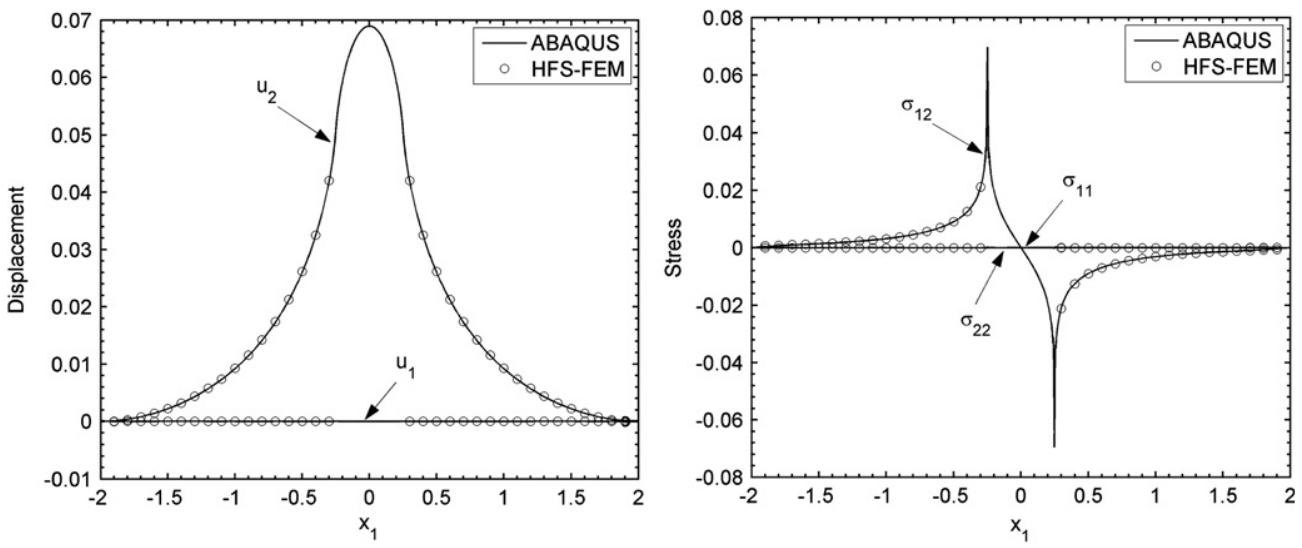

Fig. 12. Displacement and stress variations along the $x_{1}$ axis for the case of a straight line load. 
numerical results of the displacement and stress components at selected element interior points are, respectively, plotted in Figs. 9 and 10. Certainly, it would be possible to reproduce more predictions by increasing the number of element interior points within the single hybrid special element used here. As shown in the two figures, there is good agreement between the displacement and stress numerical results derived from the present method and the reference analytical solutions taken from Eqs. (55) and (56), and also it is observed that the displacement distributions are symmetric about the two coordinate axes, as we expect. More importantly, it is noted that only one 16-node special-purpose element is employed here to achieve acceptable accuracy of numerical results. In contrast, extremely refined mesh is usually required to simulate the highgradient variations of displacement and stress in the vicinity of point loads in the conventional FEM. For example, using the refined finite element mesh displayed in Fig. 8(b), including 1211 elements and 3698 nodes, the results similar to those from the proposed hybrid model can be obtained. In Table 1, analytical solutions, as well as
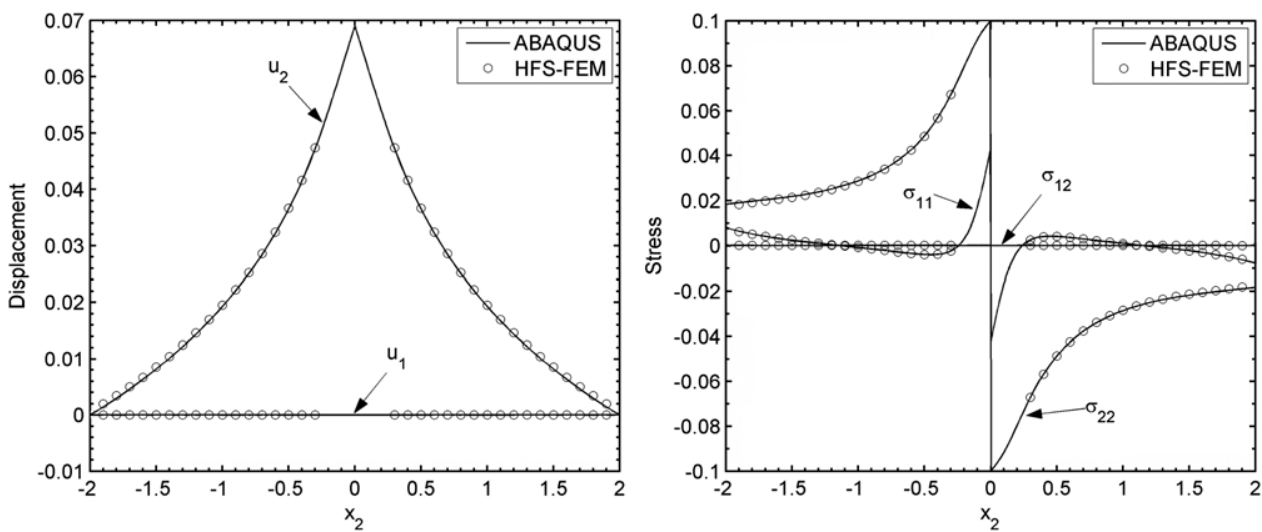

Fig. 13. Displacement and stress variations along the $x_{2}$ axis for the case of a straight line load.
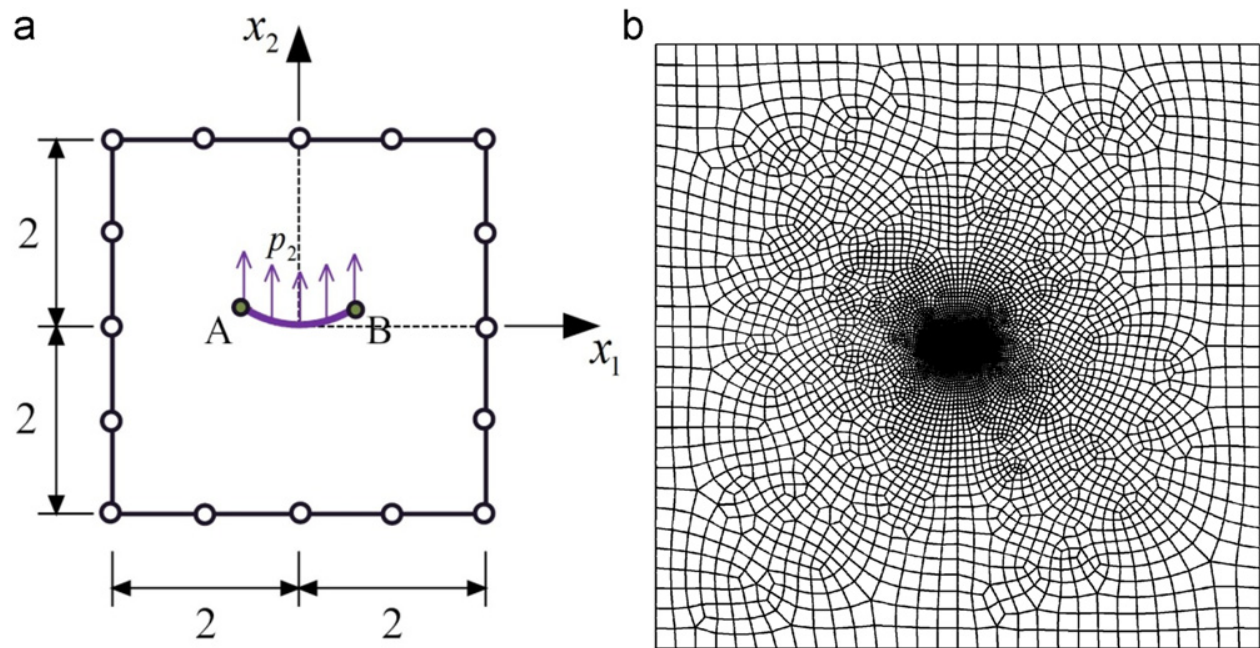

Fig. 14. (a) Sketch of square plate under curved line load and special line-load element (b) Mesh division of conventional FEM using ABAQUS.
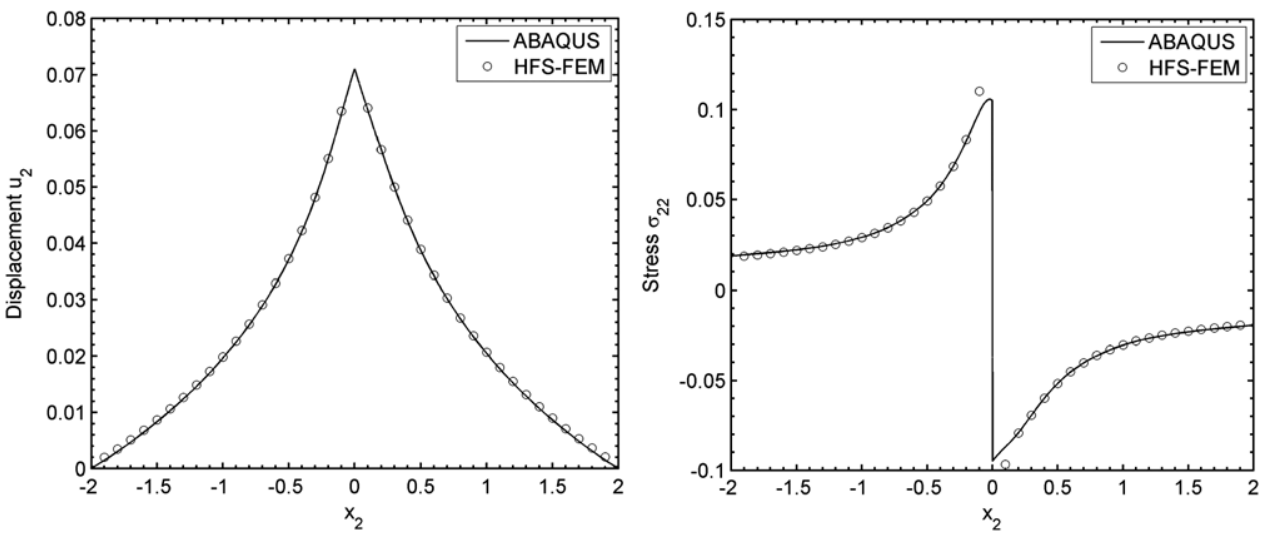

Fig. 15. Displacement and stress variations along the $x 2$ axis for the case of a curved line load. 
numerical results separately from the single hybrid model and ABAQUS at four sample points around the applied concentrated force are presented to assess the developed hybrid special element. It can be seen that the present hybrid model with just a single special element can capture the displacement and stress variation in the vicinity of the applied concentrated force more accurately than the conventional finite element model using ABAQUS with refined or coarse meshes (including 151 elements and 478 nodes). Thus, use of the special-purpose element can significantly reduce the mesh quantity and achieve desired numerical accuracy in solving such problems with local effects. Simultaneously, in the table, a refined mesh produces more accurate results than a coarse mesh in the process of $A B A Q U S$, and thus in subsequent examples in the absence of analytical solutions, numerical results from ABAQUS with extremely refined mesh are presented as reference results to assess the developed hybrid special elements.

\subsection{Local effect of line load in a square domain}

In the second example, we consider a square domain with a fixed boundary. Two types of line load are considered to assess the proposed numerical technique.

First, a horizontal straight line segment $A B$ subjected to a uniformly distributed vertical load $p_{2}=0.2$ is studied. The

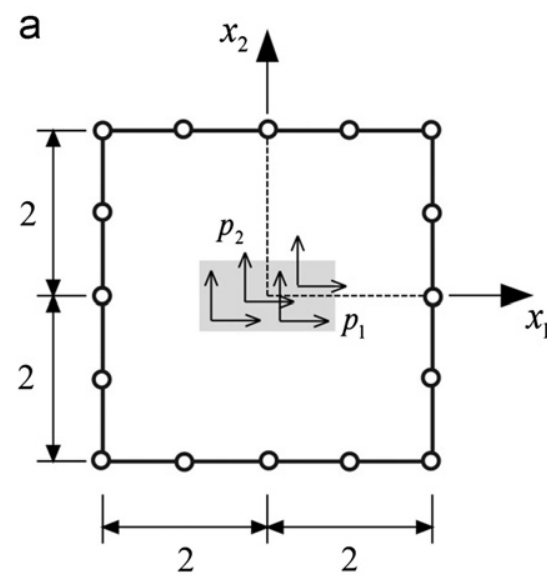

b

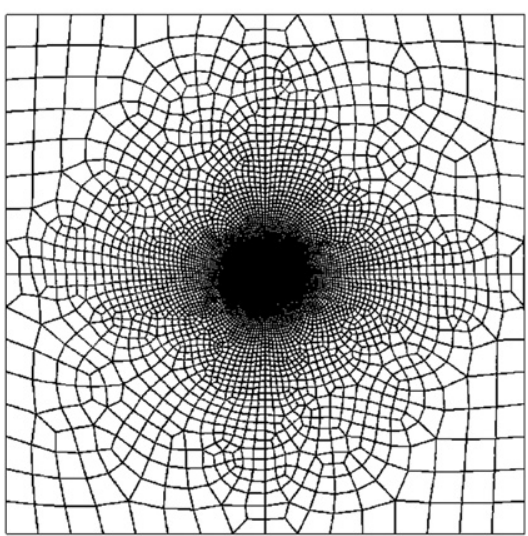

Fig. 16. Sketch of square domain under local patch load and special-purpose element (b) Mesh division of conventional FEM using ABAQUS.
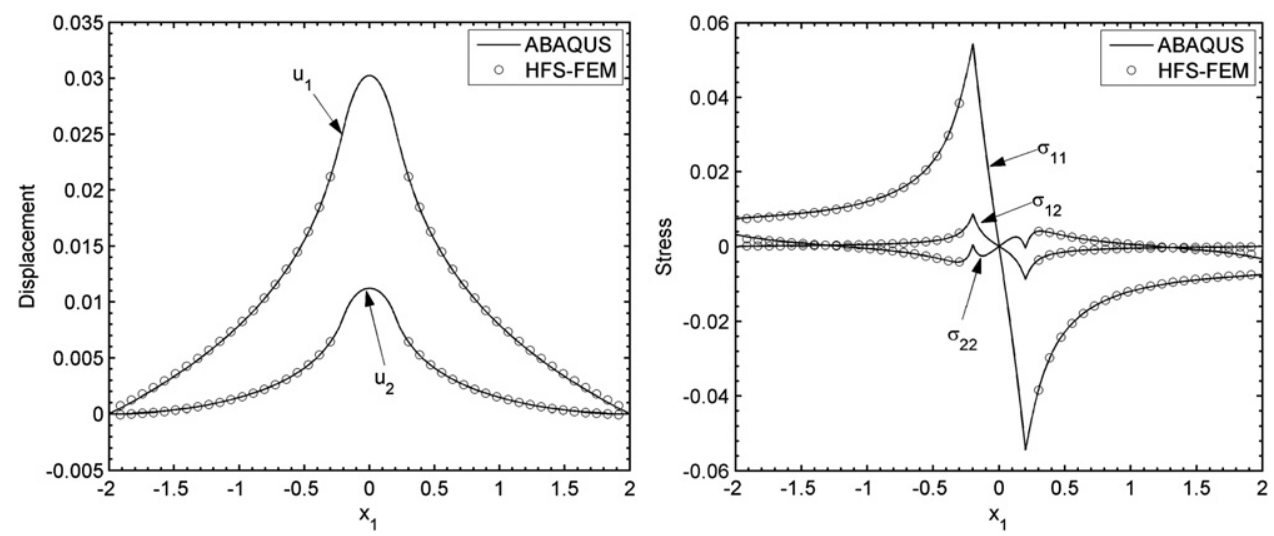

Fig. 17. Displacement and stress variations along the $x_{1}$ axis for the case of a patch load.
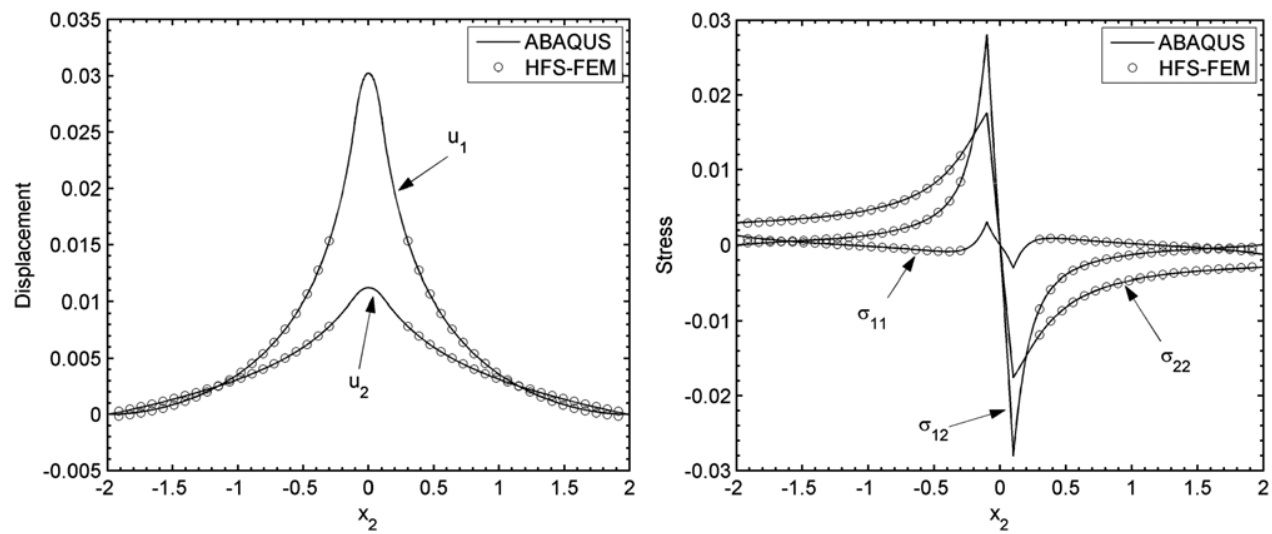

Fig. 18. Displacement and stress variations along the $x 2$ axis for the case of a patch load. 
coordinates of the two ends of the loaded line are $(-0.25,0)$ and $(0.25,0)$, respectively. Fig. 11 displays the special-purpose element mesh used in the present HFS-FEM and, for the purpose of comparison, the element discretization of the conventional FEM using ABAQUS is also provided in Fig. 11(b). In the conventional FEM, a total of 7240 plane quadrilateral elements with 21,825 nodes are employed to capture the variations of displacement and stress in the domain. Fig. 12 and Fig. 13 show the distributions of displacements and stresses along two coordinate axes, respectively. It can be seen from these two figures that the numerical solutions from the proposed formulation agree well with those from ABAQUS.

Second, a curved line segment is considered to further assess the performance of the present approach using a special-purpose lineload element. The curve is assumed to be a quadratic parabola, i.e., $x_{2}=x_{1}^{2}\left(\left|x_{1}\right| \leq 0.25\right)$. In the computation, the vertical load intensity is taken to be 0.2 and one special-purpose 16-node element is again employed to model the square domain, as shown in Fig. 14(a). The corresponding conventional FEM mesh using 7997 elements and 24,112 nodes is plotted in Fig. 14(b) for comparison. Fig. 15 illustrates the distribution of displacements and stresses. Due to the stress singularity at the origin (center point), it is observed from Fig. 15 that there is a small difference between the present HFS-FEM and ABAQUS in the numerical solutions of stress in the neighborhood of the origin. In the area which is relatively distant from the origin, however, the

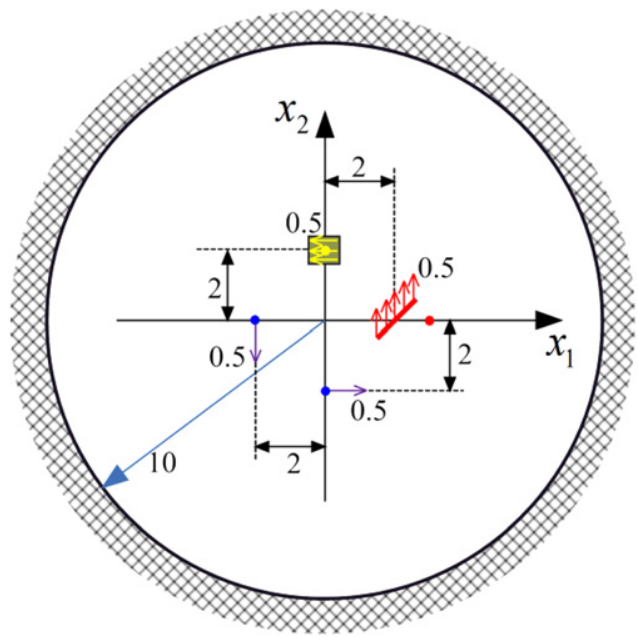

Fig. 19. Sketch of a circular disk under three types of discontinuous load. results from the proposed method agree well with those from ABAQUS.

\subsection{Local effect of patch load in a square domain}

In the third example, a rectangular patch load centered in a square domain with a fixed boundary is considered. The side lengths of the rectangle are taken as 0.4 and 0.2 . The load intensities $p_{1}$ and $p_{2}$ are assumed to be 0.5 and 0.2 , respectively. In Fig. 16, one 16-node special-purpose element is again used for the present model and 12,076 quadratic isoparametric elements are employed for ABAQUS simulation. The total number of degrees of freedom of the stiffness matrix is 32 for the proposed hybrid model and 72,586 for ABAQUS. Fig. 17 and Fig. 18 show the distributions of displacement and stress along the axes $x_{1}$ and $x_{2}$, respectively. As expected, good agreement between the results from the present hybrid finite element model and the conventional FEM is observed even though only one special-purpose element is employed in the current hybrid model. Thus the effectiveness of the proposed special patch-load element is verified in the aspect of mesh reduction.

\subsection{Circle domain under three types of discontinuous load}

To illustrate the performance of the proposed hybrid finite element model in solving complex problems, a circular disk subjected to all three types of internal discontinuous load is considered in this example (see Fig. 19). The radius of the circular disk is taken as 10 , and its rim is assumed to be fixed. The two pairs of concentrated forces, respectively, locate at $(-2,0)$ and $(0,-2)$ and the magnitude of forces is chosen to be 0.5 . The middle point of the inclined straight line load is located at $(2,0)$ and the force intensity is 0.5 . The patch load considered here is a square patch load centered at $(0,2)$ with side length 0.4 and force intensity 0.5 . A total of 144 elements with 473 nodes are employed to discretize the domain in the present approach (see Fig. 20(a)), including 140 general hybrid elements and 4 special-purpose elements. For comparison, the conventional finite element mesh generated by ABAQUS shown in Fig. 20(b) includes 26,006 elements and 78,079 nodes. Numerical results from the present method are given in Fig. 21 and Fig. 22, and good agreement is observed between the numerical solutions from the present hybrid formulation and those from the conventional FEM. a

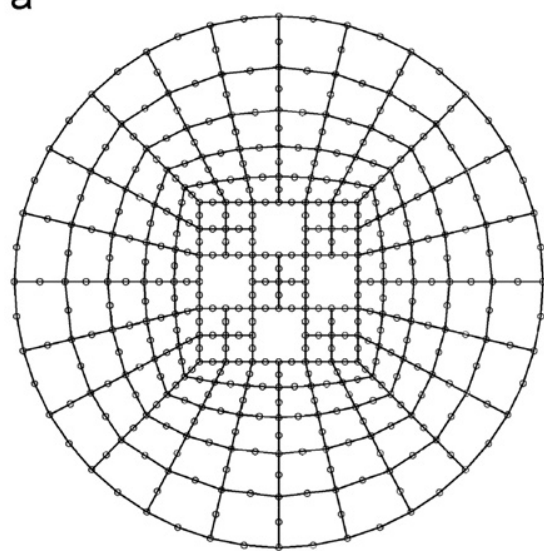

b

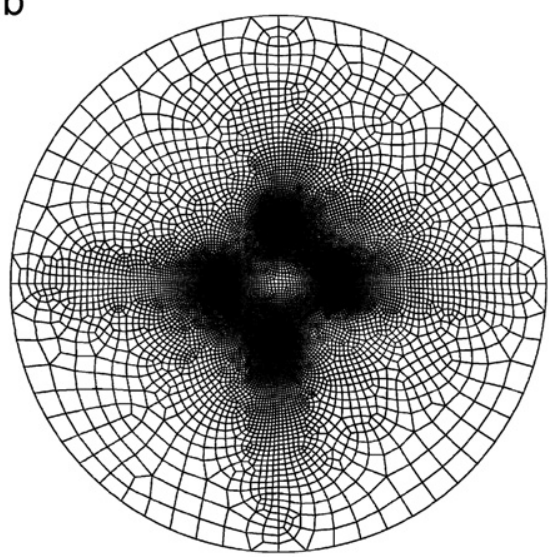

Fig. 20. Mesh divisions used in the present HFS-FEM and conventional FEM. (a) Mesh division in the present HFS-FEM and (b) Mesh division in conventional FEM. 

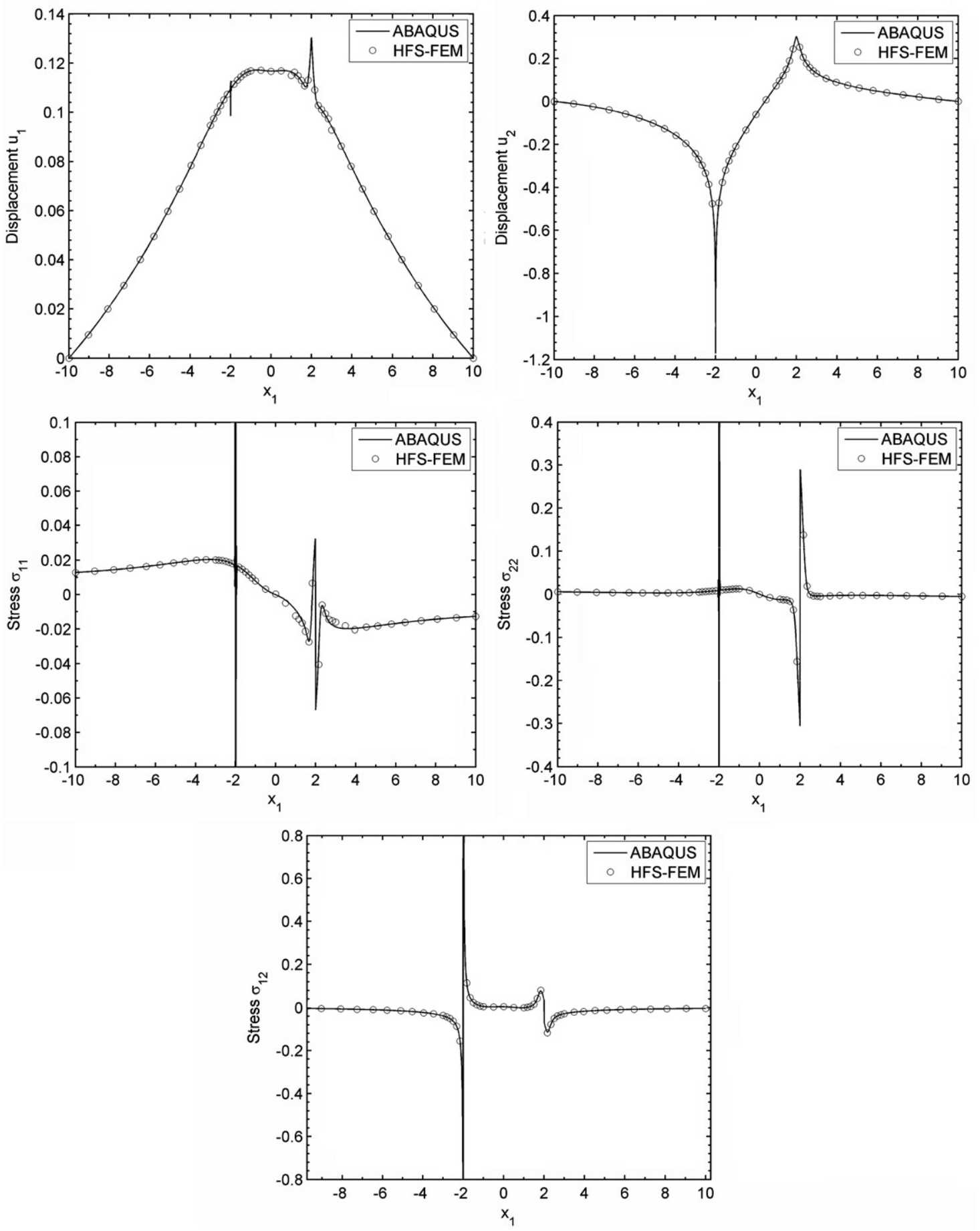

Fig. 21. Displacement and stress variation along the $x_{1}$ axis for the case of mixed loads.

\section{Conclusions}

In this paper, a boundary integral based finite element model for analyzing plane isotropic elastic solids under arbitrarily located point, line, and patch loads is presented, based on the concept of special-purpose elements. In the present formulation, the fundamental solutions of the problem are used to approximate the local effects of point, line, and patch loads as a part of the augmented particular solutions, and simultaneously, the homogeneous solutions are approximated by the linear combination of fundamental solutions at a number of source points located outside the element domain, as in the MFS. The local solutions caused by discontinuous loads are then constructed by considering them as generalized body forces. A hybrid variational functional including local effects is used to derive the corresponding boundary integral based finite element formulation and to develop special-purpose elements in which the local effects caused by discontinuous loads are involved. In particular, the problematic mesh refinement around discontinuous loads that is usually required in the conventional FEM is avoided. This advantage is highlighted through several numerical examples involving point, line, and patch loads and their combination. Numerical 

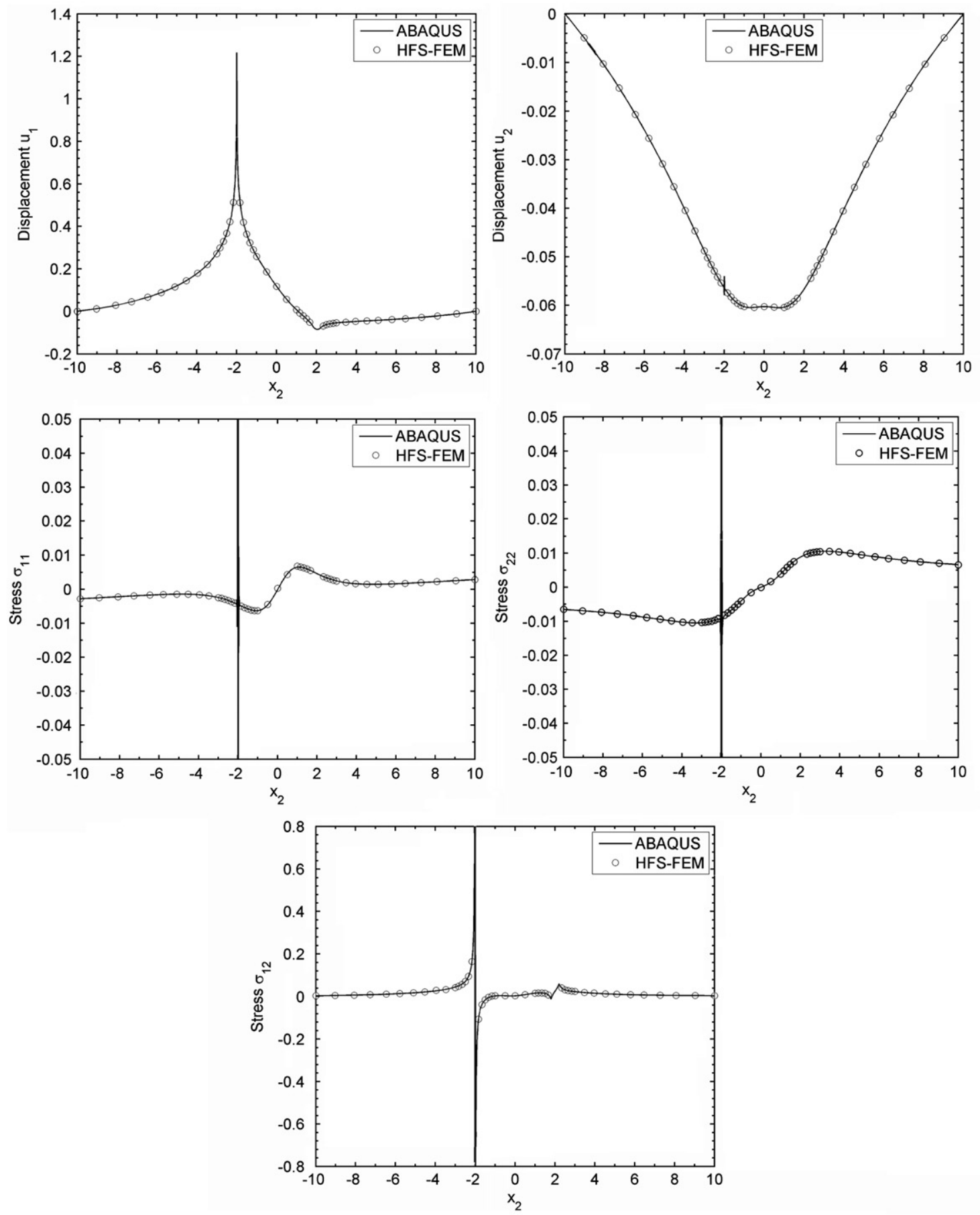

Fig. 22. Displacement and stress variations along the $x_{2}$ axis for the case of mixed loads.

results obtained using the special-purpose elements are compared with exact solutions or those from ABAQUS using a greater number of elements, illustrating the effectiveness of the specialpurpose elements in dealing with problems with interior discontinuous loads. The results show that the present model can be used to treat discontinuous loads with fewer elements.

\section{Acknowledgement}

The research in this paper is partially supported by the Natural Science Foundation of China under grant no. 11102059 and an Australian Endeavour Award 2011.

\section{References}

[1] Bathe KJ. Finite element procedures. New Jersey: Prentice-Hall, Inc; 1996.

[2] Katsikadelis JT. Boundary elements: theory and applications. Elsevier; 2002.

[3] Qin QH. Nonlinear analysis of reissner plates on an elastic-foundation by the BEM. Int J Solids Struct 1993;30(22):3101-3111.

[4] Wang H, Qin QH. Hybrid FEM with fundamental solutions as trial functions for heat conduction simulation. Acta Mech Solida Sin 2009:22(5):487-498.

[5] Wang H, Qin QH. Fundamental-solution-based finite element model for plane orthotropic elastic bodies. Eur J Mech A Solids 2010;29(5):801-809.

[6] Wang H, Qin QH. Fundamental-solution-based hybrid FEM for plane elasticity with special elements. Comput Mech 2011;48(5):515-528.

[7] Wang H, Qin QH. A new special element for stress concentration analysis of a plate with elliptical holes. Acta Mech 2012;223(6):1323-1340.

[8] Wang H, Qin QH. Boundary integral based graded element for elastic analysis of 2D functionally graded plates. Eur J Mech A Solids 2012;33:12-23. 
[9] Wang H, Qin QH. FE approach with Green's function as internal trial function for simulating bioheat transfer in the human eye. Arch Mech 2010;62(6): 493-510.

[10] Wang H, Qin QH. Special fiber elements for thermal analysis of fiberreinforced composites. Eng Comput 2011;28(8):1079-1097.

[11] Dumont NA, Lopes AAO. On the explicit evaluation of stress intensity factors in the hybrid boundary element method. Fatigue Fract Eng Mater Struct 2003;26(2):151-165.

[12] Wagner M, Gaul L, Dumont NA. The hybrid boundary element method in structural acoustics. ZAMM 2004;84(12):780-796.

[13] Venkatesh A, Jirousek J. Accurate representation of local effects due to concentrated and discontinuous loads in hybrid-Trefftz plate bending elements. Comput Struct 1995;57(5):863-870.

[14] Qin QH, Wang H. Matlab and C programming for Trefftz finite element methods. New York: CRC Press; 2008.
[15] Qin QH. Solving anti-plane problems of piezoelectric materials by the Trefftz finite element approach. Comput Mech 2003;31(6):461-468.

[16] Muskhelishvili N. Some basic problems of the mathematical theory of elasticity. Springer; 1977.

[17] Qin QH, Huang YY. BEM of postbuckling analysis of thin plates. Appl Math Modell 1990;14(10):544-548.

[18] Brebbia CA, Dominguez J. Boundary elements: an introductory course. 2nd ed. Southampton: Computational Mechanics Publications; 1992.

[19] Fairweather G, Karageorghis A. The method of fundamental solutions for elliptic boundary value problems. Adv Comput Math 1998;9(1-2):69-95.

[20] Wang H, Qin QH, Kang YL. A new meshless method for steady-state heat conduction problems in anisotropic and inhomogeneous media. Arch Appl Mech 2005;74(8):563-579. 